\title{
Introduction Special Issue Honouring Professor P. Krishnan
}

The articles included in this special issue reflect the varied research interests of Professor P. Krishnan, ranging between formal mathematical and quantitative sociological demography. Some of the authors of the works presented in this volume are former students of Krishnan's; others are long-standing colleagues who have closely followed the professional contribution of Dr. Krishnan. Although the nine papers address a wide range of topics they can be subsumed under five headings: (1) formal and applied demography (Namboodiri; Tang; Swanson); (2) Aboriginal demography (Romaniuk); (3) family change (Beaujot and Ravanera; McVey); (4) internal migration (Shin and Ram); and (5) population health (Reed; Sharma; Suwal).

Krishnan Namboodiri develops a mathematical approach devoted to the study of demographic relationships at the macro level. To accomplish this objective, Namboodiri considers aspects of vector space calculus in the context of several demographic examples, including the impact of nuptiality and fertility on the net reproduction rate and log-ratio techniques in the analysis of regression relationships involving demographic compositions, such as labour force and occupation distributions. The methods described by Namboodiri allow the researcher more flexibility in quantitative analysis of such demographic phenomena as compared to sole reliance on demographic summary measures.

The estimation of population and its main components at the local level always poses important challenges for demographers, partly because data are often unavailable or are difficult to obtain. In his paper, Zongli Tang introduces a model based on some of the classical propositions of Ravenstein, which is then applied to derive population and migration estimates for the state of Massachusetts, USA. His model assumes that local migration is determined by an area's relative attractiveness or is a compound function of distinct factors that push migrants out of the area or pull them in. The attraction factors and their change over time are measured using various data sources, including decennial census and data on group quarters and school enrollments. Tang's model is shown to yield timely population estimates whose accuracy are deemed to be superior to the corresponding estimates based on the US Census Bureau's methodology, though the author remains cautious, calling for further testing and refinements to his model. 
David Swanson's research deals with a problem not typically faced by applied demographers: the identification of a "population" using a set of criteria established by a regulatory agency. In this particular case, Swanson considers criteria established by the US Nuclear Regulatory Commission for purposes of Site Characterization of the High Level Nuclear Waste Repository proposed for Yucca Mountain, Nevada. Swanson argues that this type of task requires a wide range of skills that may become increasingly important during the course of the 21 st century. As such, budding applied demographers, especially those nearing completion of their graduate studies, will find this paper of particular interest because it points to a set of new skills that are presently not treated as part of traditional formal demographic training.

In recent years a great deal of attention has been directed to the changing demography of Canada's Aboriginal Peoples. This has become an important subspecialty of social demography. Focusing on the fertility of Canadian Aboriginals, Anatole Romaniuk outlines a history-based explanatory framework for their variant patterns of fertility as compared to the rest of Canada. Romaniuk explores several important relevant questions, including why the fertility of Aboriginals started to decline a century or so after the rest of Canada, and why in the early stages of the 21 st century Aboriginal fertility continues to surpass that of the general Canadian population by a wide margin. Romaniuk finds that of all the major social demographic theories of fertility transition, a historically based explanation provides the best fit to this specific population. He argues that both historical and contemporary fertility patterns among the Aboriginals of Canada have been strongly conditioned by the experience of culture contact with the Europeans and their consequent marginalization from mainstream society.

During the second half of the twentieth century Canada and all other high income nations have experienced profound sociological changes. As part of this general phenomenon, Rod Beaujot and Zenaida Ravanera explain that in Canada, the basis for family cohesion has shifted from organic to mechanical--or stated differently, from the breadwinner to the collaborative model. Thus, as in many other Western countries today, data on family change in Canada point to a greater flexibility for individuals in terms of their entry and exit from relationships, a delay in the timing of family events, and a diversity of family forms. Based on their analysis of these trends, a number of public policy implications are presented by Beaujot and Ravanera.

The main focus of Wayne McVey's article is on divorce and separation trends in Canada as indicators of family stability. Previous research indicated that there were an inordinate number of separated individuals in Canada as a consequence of longer required waiting periods prior to divorce. However, by 2001, the 
number of separated persons within adult age categories was far less than the number of divorced. McVey attributes this change to the introduction of more liberal divorce laws in the mid-1980s that allowed separation cases to be processed more quickly as compared to earlier times. Consequently the number of separated individuals has declined while the number of divorced persons has increased. McVey concludes his analysis by pointing out that notwithstanding high divorce rates and an increasing popularity of common law partnerships in Canada, the "traditional" family form continues to be the prevailing choice for most Canadians.

Migration is often thought as a mechanism for socioeconomic advancement. But is this always the case? Edward Shin and Bali Ram address this very question. From the Canadian evidence the answer to this question is not straightforward. It depends largely on the characteristics of migrants themselves and the characteristics of the regions of their origin and destination. Given that migration is selective of the younger, better educated and more productive workers, it is reasonable to argue that people moving from regions with less economic resources to the regions of greater resources, gain more than those who were left behind. But the story is different when migrations occur from regions with greater economic resources to the regions of less economic resources. It is also different when economy is not doing as well. During these situations, migrants need not be positively selected. People who move to economically less attractive destinations or during bad economic times are likely to be less skilled, and therefore, less likely to earn more than non-migrants.

As Canada's population continues to age, more and more attention is being devoted to the health issues and concerns of the elderly. In his investigation, Colin Reid examines the quality of care and mortality among long-term care residents with dementia across 73 long-term facilities in British Columbia. His study seeks to determine whether resident mortality within 12 months of admission to a facility can be explained by post-admission social causative factors, that is, by institutional quality of care. Reid discovered that seniors with dementia who enter long-term care facilities are actually at greater risk of death than are similar individuals that remain in the community. The implications of this finding are discussed from the point of view both further research and health policy dealing with elder care.

Raghubar Sharma looks at various social indicators to forecast child welfare caseload in the province of Ontario. Specifically he attempts to identify an optimum number of social indicators for geographical areas (i.e., census divisions) that would provide maximum predictability of child welfare caseloads. Using various regression techniques Sharma identifies ten risk factors at the census division level for child welfare caseload. 
Maternal mortality is recognised as a significant public health problem in the developing countries, most especially among the poorest nations. Juhee Suwal's investigation of maternal mortality in Nepal reveals that this type of mortality is not uniformly distributed in the female population. There are important social demographic differentials in this type of mortality. Among other factors, place of residence (whether rural or urban), region, ethnic and religious affiliation are found to correlated with mortality. Suwal concludes her analysis by listing several policy recommendations aimed at lowering maternal mortality in Nepal and in similarly poor developing countries. 